\title{
Performance of OLSR Manet Adopting CROSS-LAYER APPROACH UNDER CBR AND VBR TRAFFICS ENVIRONMENT
}

\author{
Teerapat Sanguankotchakorn ${ }^{1}$, Sanika K.Wijayasekara ${ }^{2}$ and Sugino Nobuhiko ${ }^{3}$ \\ ${ }^{1}$ Telecommunications Field of Study, School of Engineering and Technology, \\ Asian Institute of technology, Pathumthani, Thailand \\ ${ }^{2}$ Faculty of Engineering, Chulalongkorn University, Bangkok, Thailand \\ ${ }^{3}$ Interdisciplinary Graduate School of Science and Engineering, \\ Tokyo Institute of Technology, Japan
}

\begin{abstract}
The routing protocols play an important role in Mobile Ad-Hoc Network (MANET) because of the dynamically change of its topology. Optimized Link State Routing (OLSR), unawareness of Quality of Service $(Q o S)$ and power-consumed protocol, is an example of a widely-used routing protocol in MANET. The Multi-Point Relays (MPR) selection algorithm is very crucial in OLSR. Therefore, firstly, we propose a heuristic method to select the best path based on two parameters; Bit Error Rate (BER) derived from the physical layer and Weighted Connectivity Index (CI) adopted from the network layer. This can be done via the cross-layer design scheme. This is anticipated to enhance the performance of OLSR, provide QoS guarantee and improve the power consumption. The performances of the proposed scheme are investigated by simulation of two types of traffics: CBR and VBR (MPEG-4), evaluated by metrics namely Throughput, Packet Delivery Ratio (PDR), Average End-to-End Delay, Control Overhead and Average Total Power Consumption.We compare our results with the typical OLSR and OLSR using only Weighted CI. It is obvious that our proposed scheme provides superior performances to the typical OLSR and OLSR using only Weighted CI, especially, at high traffic load.
\end{abstract}

\section{KEYWORDS}

Mobile Ad-hoc Network (MANET), OLSR, Bit Error Rate (BER), Weighted Connectivity Index, Quality of Service $(Q o S)$

\section{INTRODUCTION}

MANET, an infrastructure-less mobile network, is formed by a collection of mobile interfaces without any support of the centralised administration. MANET is becoming more important in the area of wireless communication due to its self-configured nature, infrastructure-less, and its high penetration of mobile devices in the world. Due to the characteristic of the wireless network including MANET, the overall performance of this type of network is quite low [1],[2]. In addition, one of the most important constraint of mobile devices is their limited energy, the energy efficient routing becomes a main constraint in MANET environment [3],[4].

There are two main routing protocols in MANET; Proactive and Reactive protocols. In proactive routing protocol, each node creates its unique routing table by collecting the routing information broadcasted by the other nodes in the network while nodes running reactive routing protocols create their own routing table based on a request. OLSR [10] is an example of proactive protocols. And DSR (Dynamic Source Routing) and AODV (Ad Hoc On-Demand Distance- 
Vector) [7] are examples of reactive protocols. The performance comparison between proactive and reactive protocols is carried out in [35]. Most of the protocols in MANET are QoS-unaware. To provide the QoS guarantee in MANET is the challenging problem. Therefore, there is many works proposing the algorithms to provide QoS guarantee in MANET [8] [22].

This work focuses on OLSR, since it is flexible enough to support different kind of delay sensitive and multimedia applications [8],[9]. In addition, OLSR proved to be outperforming the other reactive and proactive protocols in [35]. OLSR is a QoS-unaware protocol where its characteristic is determined in terms of routing table and network coverage. OLSR consists of four main principles such as neighborhood sensing, message flooding, topology information and path computation. It has three kinds of messages namely HELLO, TC(Topology Control) and MID(Multiple Interface Declaration) [10]. HELLO messages are flooded to nearby neighbors every 2 seconds (default value). By using the information carried in HELLO messages exchanged, each node selects set of its Multipoint Relays (MPRs), a key concept in OLSR used to optimize the number of packets flooded into the network. The TC messages are forwarded by only the selected MPR nodes. The nodes create a partial topology graph based on the collected information from the flooded TC messages. A node determines the best route from source to destination based on the created partial topology graph and path computation algorithm [10].

To provide QoS, the bandwidth and delay are mainly considered in many previous works proposed in OLSR [11],[12]but they are not suitable in various scenarios where multiple QoS constraints are essential [23],[24]. The concept of Weighted Connectivity Index (CI) is firstly introduced in [13], then used to improve the OLSR performance later on [6],[8],[14],[15]. The detailed derivation of Weighted CI as well as the MPR selection method is described especially [6]. In [15], the non-additive metric such as Weighted CI and multiple additive QoS metrics in path computation method is considered. Recently, the framework called cross-layer design has proved that it can improve the performance of a wireless network, especially MANET [22],[25] [27]. The cross-layer concept is applicable in various applications, namely mobile social networks [28],[29], wireless sensor networks [26] and next generation network [22]. In terms of providing QoS guarantee in network, many works using various parameters were proposed based on the cross-layer concept [28],[30] as well. It is well known that the high packet loss as well as delay is due to the high BER in communication links [9]. In [16], the BER is considered in AODV path computation. However, the BER in proactive protocol has not been taken into account yet.

In this work, we extend the investigation further into our proposed method called CBC-OLSR [6], where the BER and Weighted CI are adopted in the cross-layer framework for OLSR protocol under two traffic types: CBR (Constant Bit Rate) and VBR (Variable Bit Rate).It is anticipated that the paths found by our proposed method have higher stability since the lowest BER and the highest Weighted Connectivity Index (CI) in a link is adopted. This results in the efficient power consumption in MANET as well. In conclusion, the main contribution of this work is the crosslayer framework using BER of the link and Weighted CI, from physical and network layer, respectively to improve the MPR-selection process and routing-table computation. This leads to more stable path between source and destination which can enhance the overall performance of OLSR as well as to reduce the overall power consumption of the network. Our proposed method is compared with standard OLSR [5],[10] and the modified OLSR proposed in [8],[13]-[15] in terms of Throughput, Packet Delivery Ratio (PDR), Average End-to-End (E2E) Delay, Control Overhead and the Average Total Power Consumption[31],[32]. In addition, the computational complexity of all three algorithms is also compared with each other.

This paper is structured as follows: Section 2 describes the related works. Section 3 describes the proposed system model as well as the detailed algorithm. Section 4 provides the detailed parameters and simulation scenarios while the results of simulation and discussion are provided in Section 5. We conclude our work in Section 6. 


\section{RELATED WORKS}

Several works to find the shortest path from source to destination were proposed in the literature [21][33]. In wireless network, especially MANET, the works on OLSR protocol have carried out mainly on the MPR selection process and routing computation. The routing computation in MANET has also been developed mainly based on shortest path algorithm.

In [21], the "look-ahead" method is improved for multi-constraint QoS routing, while an enhanced version of fully polynomial time approximation scheme for multi-constrained path optimal problem is proposed in [33]. It constructs the auxiliary graph through which the QoS parameters on each of the finding path can be guaranteed not to exceed the given constraints. Then, the nonlinear definition of path constraints is adopted to reduce both time and space complexity. The work that mainly handles the MPR selection method in OLSR is also found in the literature [18].

The new concept based on the new parameters is also proposed in [13]. The parameter called weighted CI and delay is proposed as QoS metrics in an algorithm called shortest-highest path to find the feasible paths. The research regarding finding the optimal paths using weighted $\mathrm{CI}$ is also found in [8], where the Multipoint Relaying (MPR) is modified to find the optimized paths. In [9], the MPR computation algorithm is modified to determine the lowest BER among all links between the node and the two-hop neighbors. In [34], the multipath OLSR for energy optimization is investigated. The proposed algorithm always chooses the energy-optimized path with some increase in normalized routing overhead. The work on comparison between proactive and reactive protocols can be found in [35].

In [16], the weighted CI with BER is adopted in reactive protocol AODV under cross layer design framework. It is shown under its proposed method, the performance of AODV is improved in terms of all evaluation metrics. In [15], a method called G_MCP is proposed to implement QoS routing in OLSR by considering weighted CI and multiple additive QoS. To verify that OLSR performs well for multimedia applications, the work in [36] evaluated the outcome of ad-hoc routing protocols when delivering MPEG-4 video traffic. The work about the energy consumption in MANET is carried out in, such as [3].

\section{Proposed Cross-Layer Design-based ConcePt}

In this section, we illustrate the concept of our proposed cross-layer framework called CBCOLSR, as depicted in figure 1. The Physical layer information i.e. BER, and the Application layer information i.e., QoS requirement are provided to the Network layer. The value of BER is determined by SNR of the link. Here, for simulation purpose, a model called ErrorModel 80211 was integrated into NS-2 simulator to derive the BER as given in [37].

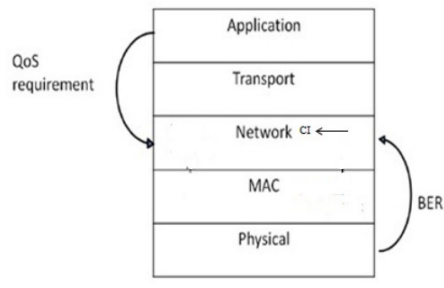

Figure 1. Proposed System Design 
Here, we modify the OLSR HELLO messages to carry the BER and one-hop weighted CI values. The MPR heuristic selection approach and the Shortest-highest path computation adopted here are illustrated in detail in section 3.3 and Figure 3. These proposed algorithms are modified from [8] and [13], respectively. Based on these algorithms, a node selects MPR nodes and the best path to a destination satisfying the lowest BER of the links. If the tie happens, then, the highest Weighted CI of the nodes will be used instead.

\subsection{Definition and Notation of Weighted CI}

The definition and detailed proof of weighted CI is covered in great detail already in [13]. Therefore, for the sake of the readers, only the necessary parts are mentioned again here. The Weighted Connectivity Index (CI) combining both node's degree and link capacity into a single metric of any network can be defined as follows:

$$
\chi_{w}=\chi_{w}(G)=\sum_{(i, j) \in E} \frac{q(i, j)}{\sqrt{d(i) d(j)}}
$$

Where $q(i, j)$ is normalized link capacity between node $i$ and $j, 0 \leq q(i, j) \leq 1$. When $q(i, j)=0$, it means the link is disconnected or unavailable.

The $n$-hop weighted CI of node $i$ can be defined as

$$
\chi_{w}\left(G_{i}^{n-h o p}\right)=\sum_{(u, v) \in E_{i}^{n-h o p}} \frac{q(u, v)}{\sqrt{d(u) d(v)}}
$$

Where $G_{i}^{n \text {-hop }}$ is a sub-graph of $G$ originating and covering up to $n$-hop from node $i$.

The weighted CI adopted here is called 2-hop Weighted CI [13] since only the information up to 2-hops is considered.

\subsection{Bit Error Rate Calculation}

Firstly, we calculate SNR using the following equation:

$$
S N R=10 \log \left(\frac{R_{x_{-} \text {power }}}{N_{r}+\sum_{i=1}^{n} R_{x_{-} \text {power }(i)}}\right)
$$

Where $R_{x \text { power }}$ denotes signal strength of the frame at the receiver which can be calculated by the propagation model, $R_{x_{-} \text {power }(i)}$ is the signal strength of frame $i^{t h}, N_{r}$ is the noise power calculated by the receiver sensitivity of the data rate used by the frame and $n$ is the total number of frames arrived.

Then, the graph between BER vs SNR provided by Intersil HFA3861B [37], as illustrated in figure 2.In this work, BPSK is considered as the modulation technique of the wireless link. 


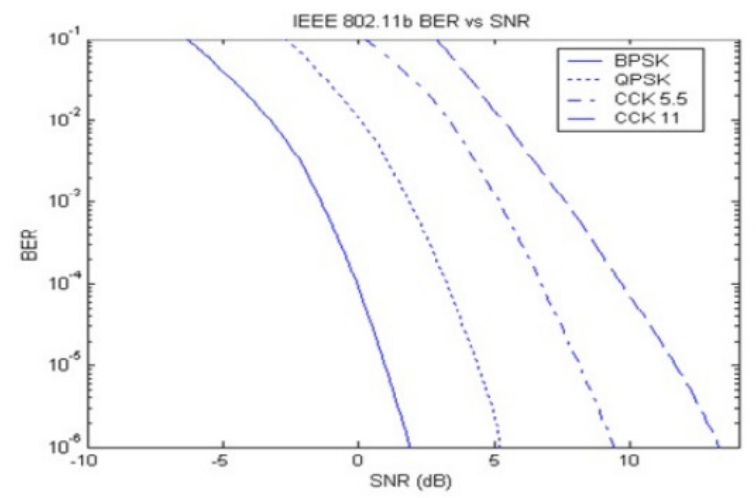

Figure 2. IEEE 802.11b BER vs. SNR [37]

\subsection{Heuristic MPR Selection Approach Based on Weighted Connectivity Index (CI) and Bit Error Rate (BER)}

The MPR selection algorithm is a crucial part of OLSR protocol since it determines the MPR nodes, which are allowed to forward the control packets over the network. Therefore, MPR selection procedure is useful to measure the quality of the OLSR protocol in terms of routing load, quality of the link in the routing table and network coverage [9]. To provide the QoS in OLSR, this research adopts the heuristic approach for MPR selection process based on minimum BER and maximum Weighted CI as illustrated as follows:

\section{Heuristic MPR Selection Process}

$\operatorname{MPR}\left(G=(V, E) ; N \_1, N \_2, M P R(x) \subset V\right)$

1. Initially, set $M P R(x)=\{\}$

2. For all nodes in $N_{-} 1$, calculate $d(y), \forall\{y\} \in N_{1}$

3. Add 1-hop neighbouring nodes in $N_{1}$ to MPR(x) to provide path to reach some nodes in $N_{2}$ 4. While the nodes in $\mathrm{N}_{2}$ exist, but are not covered by at least one node in the $\operatorname{MPR}(x)$ :

(a) Calculate the number of nodes in $N_{2}$ which are uncovered by at least one node in MPR set, for each node in $N_{l}$,

(b) Add node in $N_{1}$ that provides the lowest BER link or the highest Weighted CI to $\operatorname{MPR}(x)$

where, $M P R(x)$ is a set of neighbours of node $x$ which are selected as MPR. $N_{1}$ and $N_{2}$ are set of 1hop neighbours and 2-hop neighbours, respectively. $d(y)$ is the degree of a 1-hop neighbour of node $y$ (where $y \in N_{1}$ ).

In step 3 in this algorithm, the nodes will be declared as MPR nodes, if they are the only nodes providing the reachability to their 2-hop neighbours. While in step 4, the node having the highest weighted CI and lowest BER link to its neighbours, will be declared as MPR node by the MPR selector.

\subsection{Pseudo Code of the Proposed CBC_OLSR Algorithm}

Actually the pseudo code of our proposed CBC-OLSR algorithm is already shown in [6]. However, the detailed explanation is not sufficiently given. Therefore, the pseudo code is shown again here for the sake of explanation. In figure 3, a graph, a set of nodes and a set of links are denoted by $G, V$ and $E$, respectively where $G=(V, E)$. And $N_{1}, N_{2}$ and $T$ are the sets of 1-hop neighbors, 2-hop neighbors and topology, respectively.CBC-OLSR selects the best path based on 
the lowest BER or the highest Weighted CI value of the links. Firstly, in step 1, all entries in routing table are deleted; and all nodes in $N_{1}$ are added to routing table in step 2. Then, the hopcount, CI and BER of each link are computed. In step 3 and 4, all nodes in $N_{2}$ and/or $T$ will be added to routing table if there is no path to reach destination stored in routing table; or it provides a better path in term of higher value of CI and lower value of BER. In step 4, the BER of all paths found will be computed and compared to find the minimum one. If the tie occurs, then, the algorithm will select the path based on the highest Weighted CI and declare it in the routing table.

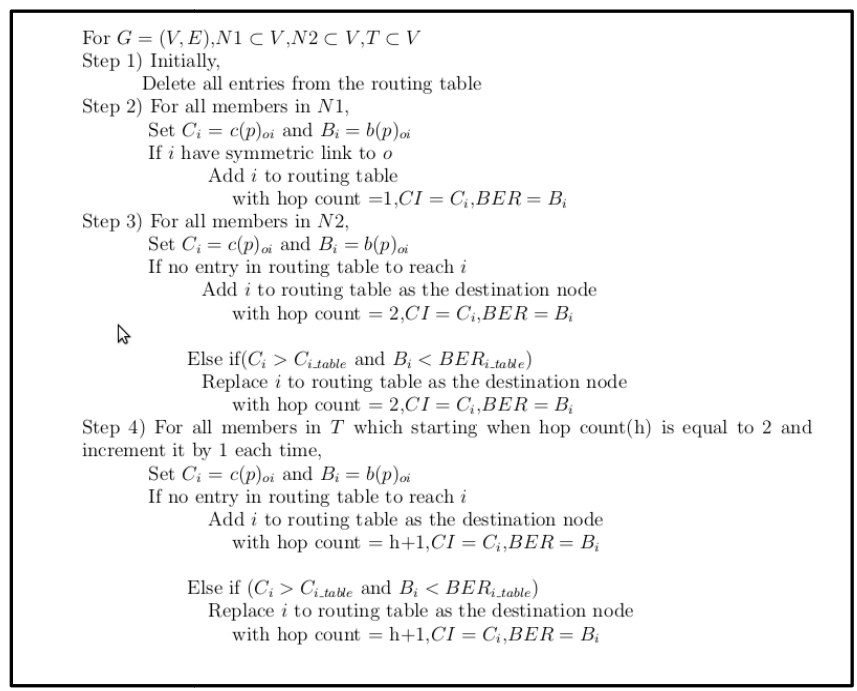

Figure 3. Modified Shortest-highest Path Algorithm

For the sake of the readers, the example illustrating the proposed algorithm is provided as follows:

Example: Consider the network topology shown in figure 4. Assume that all links Weighted CI and BER are known and illustrated in the form of (Weighted CI, BER), where Weighted CI and BER in this example are in arbitrary unit. The detailed calculation of Weighted $\mathrm{CI}$ can be found in [13]-[15], therefore, we skip it here.

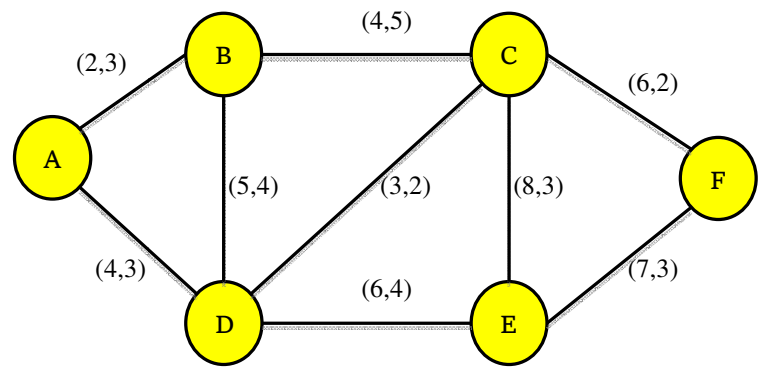

Figure 4. Example Network Topology

Based on the algorithm illustrated in figure 3, assume that we need to find the best path from Node A to F. Firstly, link A-D is selected since it has the higher Weighted CI than link A-B, while both have the same BER. Next, link D-E is selected since it has the highest Weighted CI among all links connecting to node D. Similarly, the link E-D is selected. Finally, link C-F is chosen. That is, the higher priority is given to the Weighted CI if the tie of BER occurs. 
International Journal of Computer Networks \& Communications (IJCNC) Vol.10, No.6, November 2018

\section{Simulation Parameters and Performance Evaluation Metrics}

\subsection{Simulation Parameters}

This section illustrates the parameters and the performance evaluation metrics used in our simulations for both CBR and VBR (MPEG-4) services. The parameters used in our simulations are listed in table 1, while table 2 illustrates the power consumption in each node's state [38] used in our simulations of the energy consumption of the network.

The definition of power consumption of each state is as follows:

- Transmit: node transmits a packet with this transmission power.

- Receive: node consumes this amount of power when receiving a packet regardless of correct, erroneous or garbled reception.

- Idle: when no packet is received, nor transmitted, node keeps listening to the medium and consumes this amount of power. We assume that nodes can change from "Idle" state to "Transmit" or 'Receive" state immediately without any power consumption in transition period.

- Sleep: the node becomes "Sleep" state if the residual power of node is lower than the minimum power in "Idle" state. Within this state, the node cannot detect any signals because the radio is switched off.

Table 1. Simulation Parameters.

\begin{tabular}{|c|c|c|c|c|}
\hline \multirow[t]{2}{*}{ Parameters } & \multicolumn{2}{|c|}{ CBR } & \multicolumn{2}{|c|}{ MPEG-4 } \\
\hline & $\begin{array}{c}\text { Node } \\
\text { Density }\end{array}$ & $\begin{array}{c}\text { Node } \\
\text { Mobility }\end{array}$ & $\begin{array}{c}\text { Node } \\
\text { Density }\end{array}$ & $\begin{array}{c}\text { Node } \\
\text { Mobility }\end{array}$ \\
\hline Area $\left(m^{2}\right)$ & \multicolumn{4}{|c|}{$1,000 \times 1,000$} \\
\hline Link Capacity $(M b p s)$ & \multicolumn{4}{|c|}{2} \\
\hline $\begin{array}{l}\text { Number of Nodes (Number } \\
\text { of Connections) }\end{array}$ & $\begin{array}{l}10(2)- \\
50(10)\end{array}$ & $50(10)$ & $\begin{array}{l}10(2)- \\
50(10)\end{array}$ & $50(10)$ \\
\hline Speed $(\mathrm{m} / \mathrm{s})$ & 2 & $1-30$ & 2 & $1-30$ \\
\hline Pause Time $(s)$ & \multicolumn{4}{|c|}{0} \\
\hline Video Source & \multicolumn{2}{|c|}{-} & $\begin{array}{l}\text { Foreman } \\
(176 \times 144)\end{array}$ & QCIF \\
\hline Packet Size (bytes) & \multicolumn{2}{|c|}{100} & \multicolumn{2}{|c|}{1,500} \\
\hline $\begin{array}{l}\text { Traffic } \quad \text { Rate/Connection } \\
(K b p s)\end{array}$ & \multicolumn{2}{|c|}{100} & \multicolumn{2}{|c|}{$200-400$} \\
\hline Mobility Model & \multicolumn{4}{|c|}{ Random Way Point } \\
\hline $\begin{array}{l}\text { Node Transmission Range } \\
(\mathrm{m})\end{array}$ & \multicolumn{4}{|c|}{250} \\
\hline Simulation Time $(s)$ & \multicolumn{4}{|c|}{300} \\
\hline No. of Executions/Scenario & \multicolumn{4}{|c|}{20} \\
\hline
\end{tabular}

Table 2. Power Consumption in each Node's state

\begin{tabular}{|c|c|}
\hline State & Power Consumption $(\boldsymbol{W})$ \\
\hline Transmit & 1.3 \\
\hline Receive & 0.9 \\
\hline Idle & 0.74 \\
\hline Sleep & 0.047 \\
\hline Initial Energy $(J)$ & 1,000 \\
\hline
\end{tabular}




\subsection{Performance Evaluation Metrics}

Our proposed CBC-OLSR algorithm is evaluated using the following evaluation metrics:

- Throughput: the amount of data successfully transmitted over the network in a unit of time.

- Packet Delivery Ratio (PDR): the ratio of received packets at destination with respect to the total number of packets transmitted by sources.

- Average End-to-End Delay (E2E):the mean value of the overall delay experienced by all packets under consideration.

- Control overhead: the total number of non-data packets transmitted within the network.

- Average Total Power Consumption: the average of all energy consumed by all nodes in the network in a period under consideration.

\section{SiMUlation RESUltS AND DisCUSSION}

In this section, the simulation results of our proposed CBC-OLSR algorithm are compared with standard OLSR and OLSR using only Weighted CI algorithm. All simulations are carried out using NS2 simulator [39]. The traffics under consideration in this work are CBR and VBR (MPEG-4 video).The performances are analysed using the afore-mentioned performance evaluation metrics. We run the simulations 20 times per one data point and all the results are illustrated with $95 \%$ confidential interval to ensure the validity of the simulation.

\subsection{Constant Bit Rate Service}

\subsubsection{CBR: Effect of Node Density}

This section shows the impact of node density on the performance evaluation metrics of our proposed CBC-OLSR, standard OLSR and OLSR using only Weighted CI. Actually, we carried out the simulations on various speed, but, we illustrate the results at only speed $=2 \mathrm{~m} / \mathrm{s}$ due to the same trend of results for the other speeds.

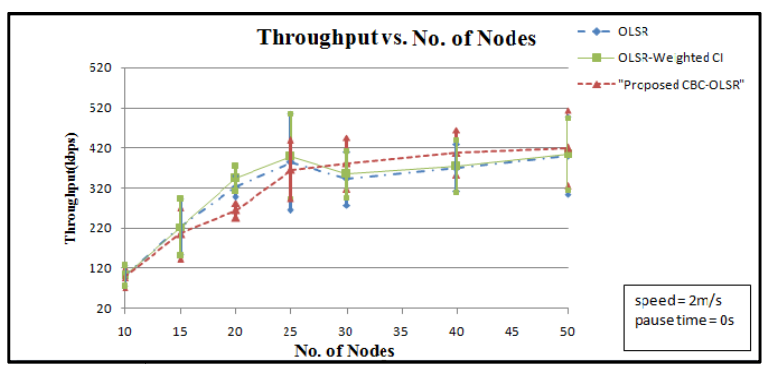

Figure 5. CBR: Throughput vs. Number of Nodes

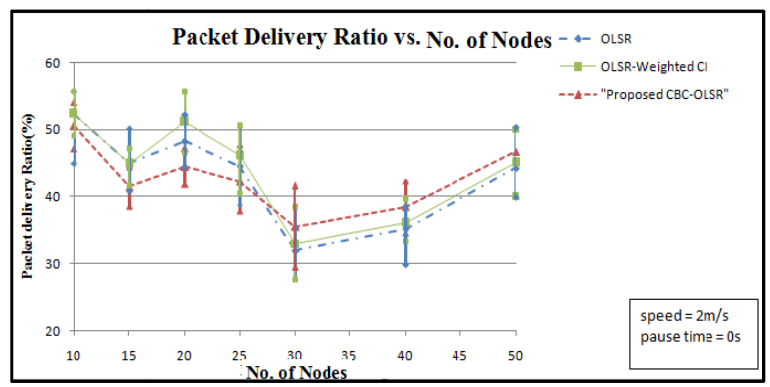

Figure 6. CBR: Packet Delivery Ratio vs. Number of Nodes 


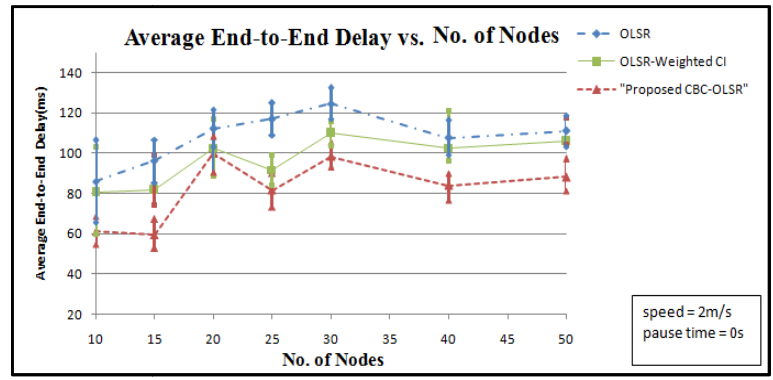

Figure 7. CBR: Average End-to-End Delay vs. Number of Nodes

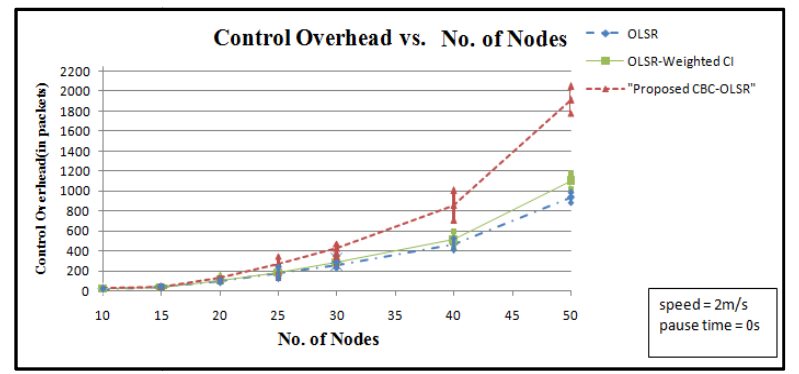

Figure 8. CBR: Control Overhead vs. Number of Nodes

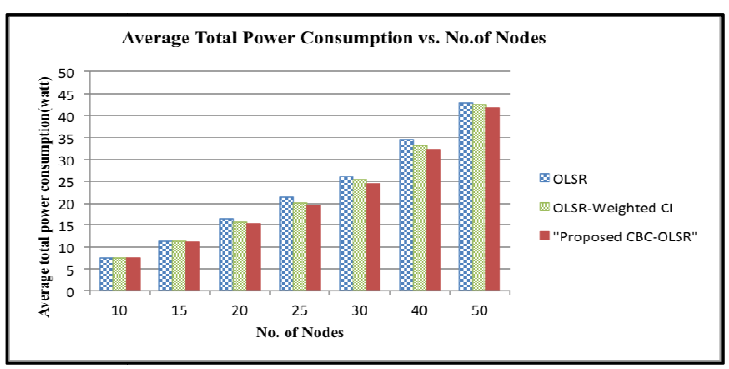

Figure 9. CBR: Average Total Power Consumption vs. Number of Nodes

Figures 5 7 illustrates the Throughput, PDR and Average E2E Delayers us number of nodes. It is obvious that our proposed CBC-OLSR provides the best performance when the number ofnodes is larger than 30. This is due to the best path can be identified by the MPR nodes selected based on our proposed algorithm. The path identified is the best due to the highest stability (minimum BER and maximum weighted CI).

When the number of nodes in the network is small (less than 30), in general, the average transmission distance between nodes become longer as well as the node's degree becomes smaller which results in higher BER and frequent link broken. While in standard OLSR, the MPR nodes and best path are selected based on only the number of hop-count whereas, in OLSR with only Weighted CI, the best path is selected based on only the highest Weighted CI where the BER is not put into account. Therefore, the path found based on minimum BER and maximum Weighted CI is probably hard to find or, even though it is found, broken frequently. This results in lower Packet Delivery Ratio and Throughput in our proposed algorithm comparing to the other two algorithms. However, the path found by our proposed CBC-OLSR is still the most stable path, which can provide the lowest Average E2E Delay among all algorithms. 
When the number of nodes increases, the average transmission distance between the nodes decreases. Generally, this results in lower BER in transmission links and also increases the degree of neighbours around a node, which reduces the link loss. This results in higher throughput and PDR but lower average end-to-end delay in our proposed CBC-OLSR.

Figure 8 illustrates the comparison of Control Overhead of three algorithms. It is apparent that the Control Overhead of all algorithms increases exponentially with the increment of number of nodes. It is apparent that the control overhead of our proposed CBC-OLSR is the highest, because of the increasing number of MPR nodes introduced by our proposed MPR heuristic algorithm.

Figure 9 illustrates Average Total Power Consumption of all algorithms. The Average Total Power Consumption increases with the increasing number of nodes. It is obvious that our proposed CBC-OLSR consumes lowest energy among three algorithms. Since our proposed CBC-OLSR algorithm selects the most stable routes based on minimum BER and maximum Weighted CI. Therefore, it can reduce the effect of interference and medium collisions, which leads to the link failures between the nodes in a network. This results directly in the reduction of the total power consumption in transmitting and receiving packets in the network.

Even though the Control Overhead of our proposed method is the highest among three algorithms, however, its size is very small comparing to data packet's size. Therefore, even the CBC-OLSR algorithm issues the highest number of Control Overheads; still the power consumption is lowest due to the stability of the selected routes.

\subsubsection{CBR: Effect of Node Mobility}

In this scenario, we investigate the performance of all algorithms in various nodes' speed environment.

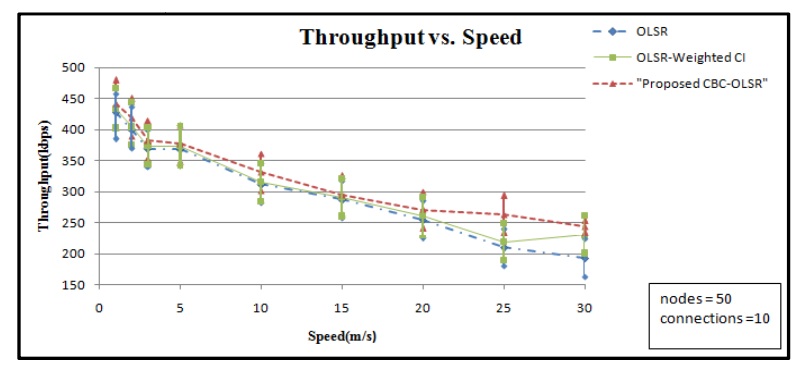

Figure 10. CBR: Throughput vs. Speed

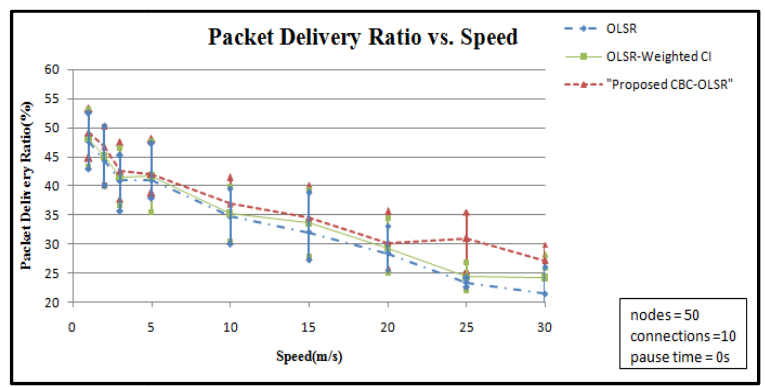

Figure 11. CBR: Packet Delivery Ratio vs. Speed 


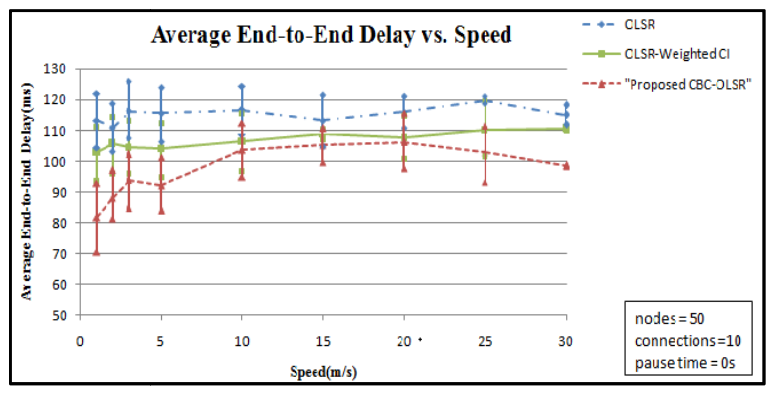

Figure 12. CBR: Average End-to-End Delay vs. Speed

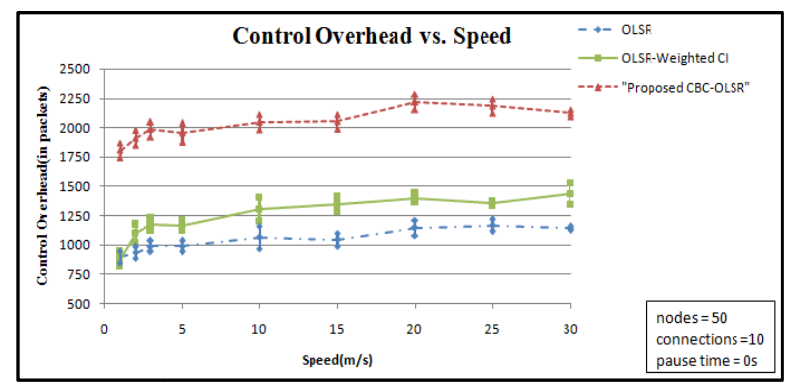

Figure 13. CBR: Control Overhead vs. Speed

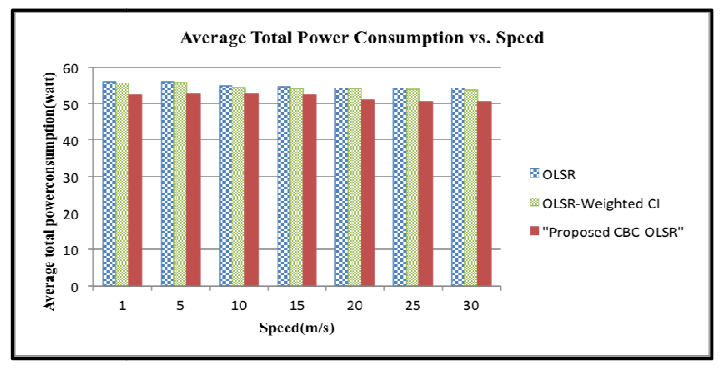

Figure 14. CBR: Average Total Power Consumption vs. Speed

As shown in figures 10 12, it is obvious that our proposed CBC-OLSR provides the highest Throughput and Packet Delivery Ratio and the lowest Average End-to-End Delay among three algorithms at all speed under consideration here. Because the path selected by our proposed is based on minimum BER and maximum Weighted CI, therefore, this path is supposed to be the most stable path. However, the probability of link-break increase with the increment of nodes' speed, this results in performance degradation of Throughput, PDR and Average E2E Delay.

In figure 13, our proposed CBC-OLSR has the highest number of Control Overhead in all speed while standard OLSR has the lowest one. Since the standard OSR uses native MPR selection method to optimize the number of Control Overhead while OLSR with only weighted CI has to consider the maximum Weighted CI of the nodes in the MPR selection method, whereas, in our proposed CBC-OLSR, it has to consider both the minimum BER of the links and the maximum Weighted CI.

As depicted in figure 14, when the speed increases the Average Total Power Consumption lightly decreases. According to the energy model [32], the energy consumption in transmitting/receiving of data packets is mainly depending on the size of data packets. Since the size of MAC or routing 
control packet is very small comparing to the size of data packet, therefore, the power consumption in any nodes can be approximated by the power consumption of only data packets. Hence, when the speed of nodes increases, the amount of data packets flooded over the network (as depicted as Throughput in figure 10) become lower due to the frequent occurrence of link break. This results in the decrement of power consumption of all algorithms. As discussed earlier, the path found by our proposed algorithm is more stable than those of the other ones; therefore, the loss due to link break is small. This result in the least Total Power Consumption of our proposed CBC-OLSR among all algorithms considered here.

\subsection{Variable Bit Rate (VBR: MPEG-4) Services}

The MPEG-4 video traffic is generated by integrating EvalVid toolset with NS-2 and usingthe Foreman YUV QCIF (176X144) as the video source with 400 frames [40].

\subsubsection{MPEG-4: Effect of Node Density}

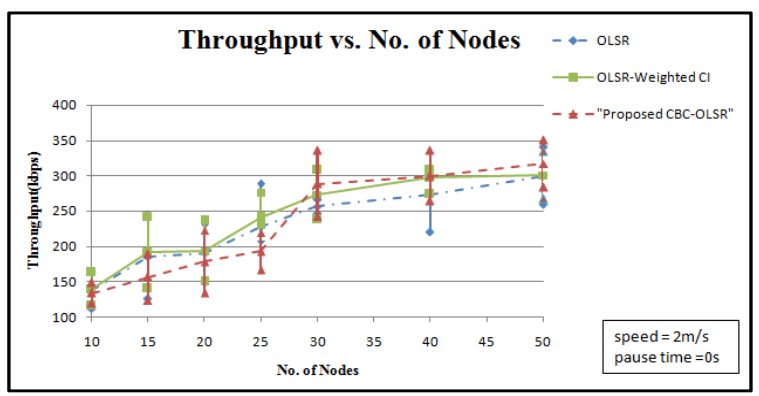

Figure 15. MPEG-4: Throughput vs. Number of Nodes

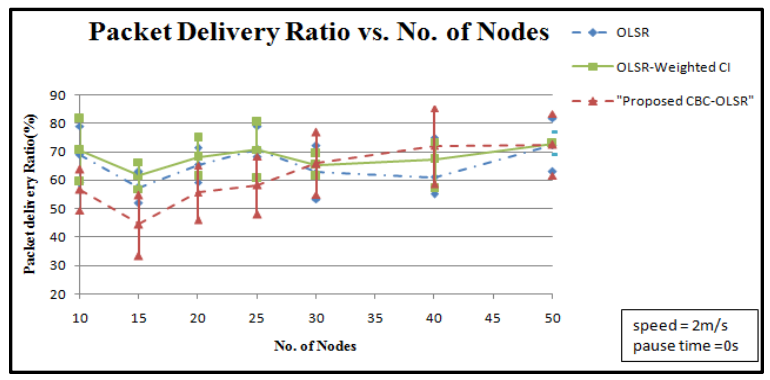

Figure 16. MPEG-4: Packet Delivery Ratio vs. Number of Nodes

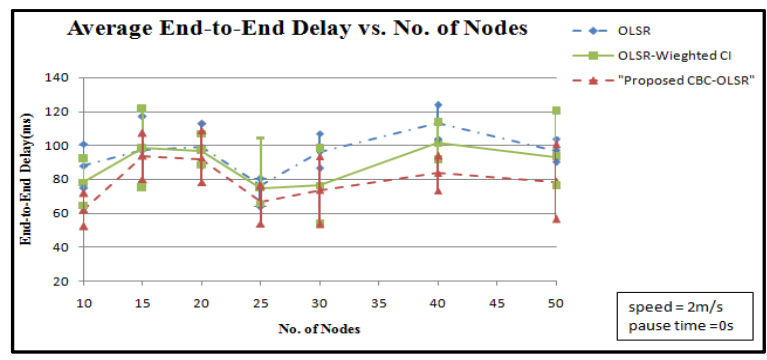

Figure 17. MPEG-4: Average End-to-End Delay vs. Number of Nodes 


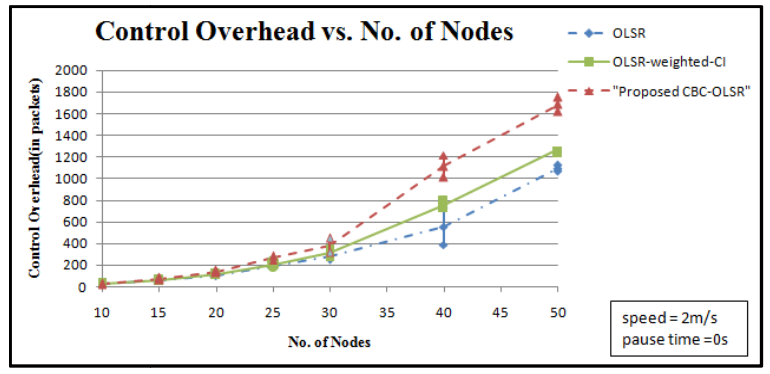

Figure 18. MPEG-4: Control Overhead vs. Number of Nodes

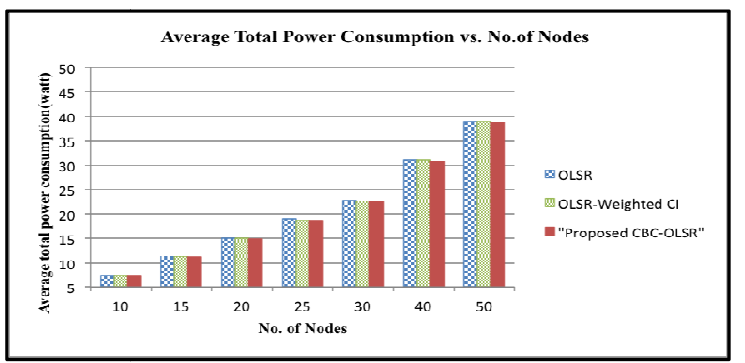

Figure 19. MPEG-4: Average Total Power Consumption vs. Number of Nodes

It is apparent, as shown in figures 15 16, that our proposed CBC-OLSR is the most superior among all three algorithms at a large number of nodes. The reason is similar to the CBR service case.

As shown in figure 17, the proposed CBC-OLSR depicts the lowest Average E2E Delay among three algorithms. It can be noticed that all algorithms comply with the E2E delay requirement recommended in ITU G.114 [41] recommendation.The Average E2E2 Delay slightly increases at high node density because of the increment of control packets. Figure 18 illustrates that our proposed CBC-OLSR generates the highest number of Control Overheads into the network because a large number of Hello and TC packets are needed to collect the nodes degree and sense the SNR (in order to calculate BER).

As depicted in figure 19, when the number of nodes increases, the Total Power Consumption also increases due to the large amount of traffic flooded into the network. As mentioned previously, our proposed CBC-OLSR provides more stable route, which reduces the probability of link failures in the network. This results in the increment of network lifetime by saving the battery power of mobile nodes in MANET. Therefore, the proposed CBC-OLSR consumes the lowest power among three algorithms even though higher Control Overheads are flooded into the network. As mentioned previously, the size of control packet is very small comparing to the size of data packet; therefore, the power consumption due to the control packets does not affect the Total Power Consumption in the network. 


\subsubsection{MPEG-4: Effect of Node Mobility}

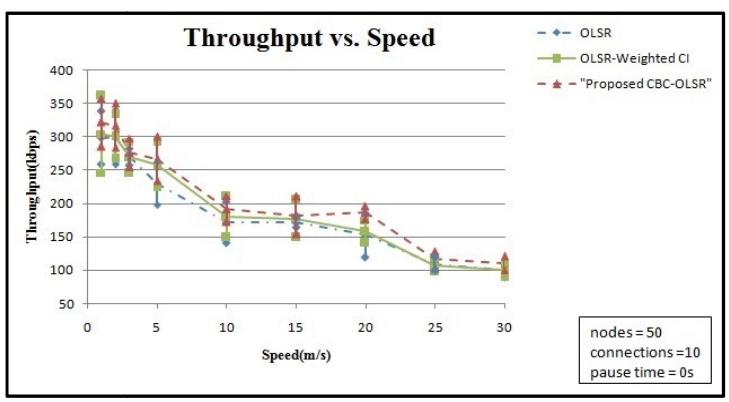

Figure 20. MPEG-4: Throughput vs. Speed

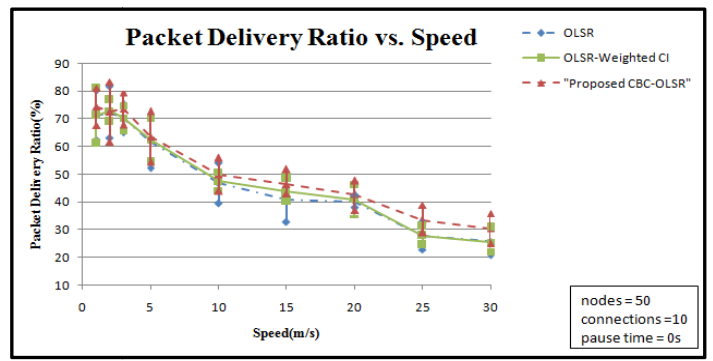

Figure 21. MPEG-4: Packet Delivery Ratio vs. Speed

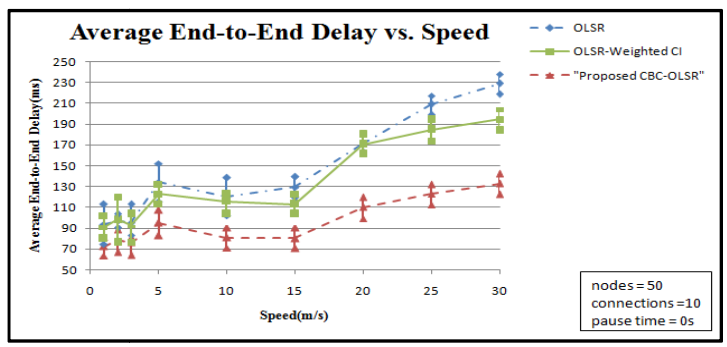

Figure 22. MPEG-4: Average End-to-End Delay vs. Speed

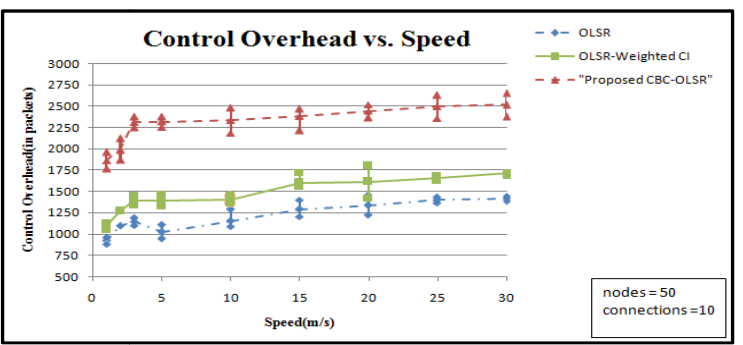

Figure 23. MPEG-4: Control Overhead vs. Speed 


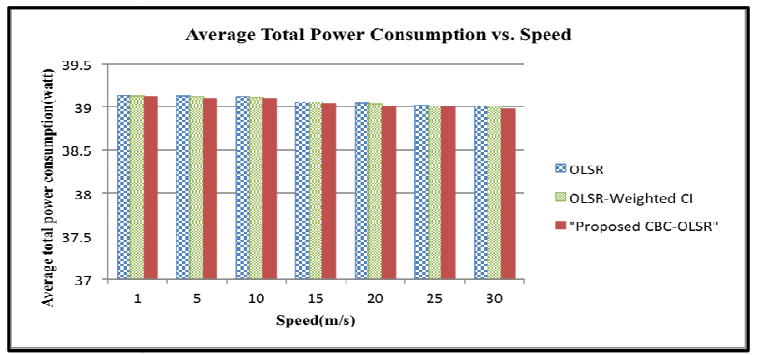

Figure 24. MPEG-4: Average Total Power Consumption vs. Speed

Figures 20 22 illustrate the simulation results of Throughput, PDR and Average E2E Delay versus speed. It is apparent that the overall performance of all three metrics degrades when the speed increases. Regarding the Throughput and PDR, it is obvious that our proposed CBC-OLSR is a little bit superior to the other two algorithms in all speeds. Since the proposed algorithm selects the MPR nodes and the best path based on the lowest BER and the highest Weighted CI. Therefore, the path selected is more stable than those of the other two algorithms. This results in higher Throughput and Packet Delivery Ratio. The OLSR only with Weighted CI performs a little better than standard OLSR, since it considers bandwidth in its path selection process.

Our proposed CBC-OLSR provides the lowest Average E2E Delay among all three algorithms at all speed, as shown in figure 22. It is obvious that only our proposed method provides the Average E2E Delay satisfying the E2E delay recommendation at high speed in ITU G.114 [5], which requires the one-way E2E delay to be less than $150 \mathrm{~ms}$. Moreover, when the speed increases, the link break occurs more frequent. This results in the increment of Average E2E Delay and Control Overhead in all three algorithms.

As illustrated in figure 23, our proposed algorithm provides the highest number of Control Overheads among three algorithms due to the need of Hello and TC messages generated to collect the nodes' degree and the SNR. However, the standard OLSR has the lowest number of Control Overheads because of the optimization of the number of MPR selected as well as the number of Control Overhead flooded into the network.

As shown in figure 24, due to the stable route selected based on the lowest BER and the highest Weighted CI, the proposed algorithm provides a little bit lower Total Power Consumption than standard OLSR and OLSR only with Weighted CI algorithms in high mobility environment. This can be explained by the same reason as the case of CBR service.

\subsection{Computational Complexity}

To study and compare the Computational Complexity of the proposed CBC-OLSR with standard OLSR and OLSR with only Weighted CI, we run the simulation of all algorithms on the exactly same platform and environment as shown in table 3 . Then, the execution time is measured and used indirectly as a measurement of Computation Complexity of the algorithms. The simulation result is illustrated in figure 25 . 
Table 3. Simulation Platform and Environment

\begin{tabular}{|c|c|}
\hline Parameters & Value \\
\hline Simulator & NS 2 Version 2.34 \\
\hline Operating System & 32 bit Ubuntu 11.10 (oneiric) \\
\hline Processor & Intel core i3, 2.3 GHz \\
\hline Memory $(\mathrm{GB})$ & 2 \\
\hline Simulation Time $(s)$ & 60 \\
\hline
\end{tabular}

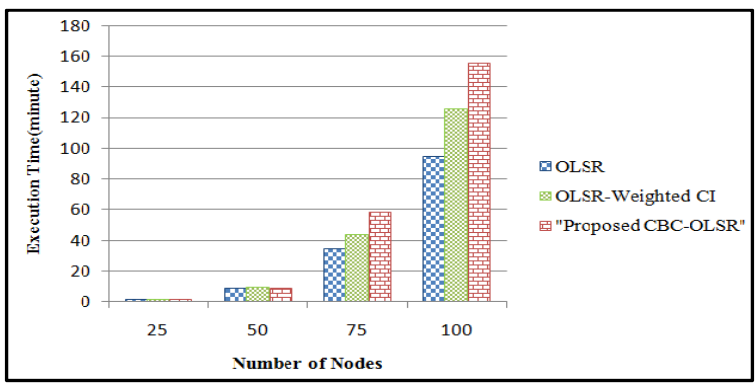

Figure 25. Execution Time vs. Number of Nodes

As shown in figure 25, it is obvious that the execution time of the proposed CBC-OLSR is the highest among all three algorithms, especially at the high number of nodes. When the number of nodes is small, the computation time may be negligible. However, it becomes significant when the number of nodes is large due to the drastically increasing computation of both BER and Weighted CI.

\section{Conclusions}

In this work, we extend the investigation of our proposed cross-layer framework for OLSR called CBC-OLSR. The proposed CBC-OLSR algorithm selects the MPR nodes and best routes in Network layer based on the BER and Weighted CI parameters.

We perform various simulations under different node density, speed and traffic types; CBR and VBR (MPEG-4 video). The performance comparison between our proposed CBC-OLSR, standard OLSR and OLSR with only Weighted CI is shown. We observe that the proposed CBCOLSR performs well in both high node density and various speed conditions for both CBR and VBR traffics. Moreover, it is obvious that our proposed algorithm consumes the lowest power among all algorithms studied here at all speed and node density. Since it selects the most stable path from source to destination based on minimum BER and maximum Weighted CI. This reduces the occurrence of link break in transmitting/receiving packets between source and destination. Hence, it improves the overall performance including the power consumption of OLSR. Consequently, it can be concluded that our proposed CBC-OLSR is an efficient algorithm, which has the capability to send data over the network and also saves the overall battery power of mobile units in MANET. However, regarding the computational complexity, it is observed that our proposed algorithm has the highest complexity, especially at high node density due to the computation of BER of each link as well as Weighted CI of each node.

\section{ACKNOWLEDGEMENTS}

This work is partially supported by National Institute of Information and Communication Technology (NICT), Japan. The authors would like to express their sincere thanks here. 
International Journal of Computer Networks \& Communications (IJCNC) Vol.10, No.6, November 2018

\section{REFERENCES}

[1] Hui-Hsiang Khao, Peng-Jung Wu, Chung-Nun Lee, "Analysis and Enhancement of Multi-channel MAC Protocol for Ad Hoc Networks", International Journal of Communication Systems, Vol. 24, pp. 310-324, 2011.

[2] Rony Ohayon, "Virtual Reservation Scheme for Supporting CBR Multimedia Services with Strict QoS Performance over WLAN and Wireless Mesh", International Journal of Communication Systems, Vol.25, pp.571-584, 2012.

[3] Saoucene Mahfoudh and Pascale Minet, "An Energy Efficient Routing Based on OLSR in Wireless Ad Hoc and Sensor Networks", The 22nd IEEE International Conference on Advanced Information Networking and Applications, IEEE Computer Society,pp.1253-1259, 2008.

[4] Ren-Hung Wang, Chiung-Ying Wang, Chi-Jen Wu and Guan-Nan Chen, "A Novel Efficient Powersaving MAC Protocol for Multi-hop MANETs", International Journal of Communication Systems, Vol.26, pp.34-55, 2013.

[5] Y.Chen, T.Farley and N.Yel, "QoS Requirements of Network Applications on the Internet", Information-Knowledge-Systems Management, pp.55-76,2004.

[6] T. Sanguankotchakorn, Sanika K. Wijayasekara and Nobuhiko Sugino, "A Cross-layer Design Approach in OLSR MANET using BER and Weighted Connectivity Index", The 19th IEEE International Conference on Networks (ICON2013), Orchard Hotel, Singapore, pp.1-6, December 11th-13th, 2013.

[7] C.E.Perkins and E.M.Royer: "Ad-hoc On-demand Distance Vector Routing", The 2nd IEEE Workshop on Mobile Computing System and Applications (WMCSA), pp.99-100, February 1999.

[8] K.Kunavut and T. Sanguankotchakorn: "Optimized Path Selection Process in OLSR Based on Weighted Connectivity Index and Delay", The 8th International Conference on Electrical Engineering/Electronics, Computer, Telecommunications and Information Technology (ECTI-CON 2011), pp. 348-351, May 2011.

[9] T.Yelemou, P.Meseure and A.-M. Poussard: "A new BER-based Approach to improve OLSR Protocol", The 8th International Conference on Wireless and Optical Communications Networks (WOCN 2011), pp. 1-5, May 2011.

[10] T. Clausen and P. Jacque: "Optimized Link State Routing Protocol (OLSR)",Technical Report ,RFC3626, IETF Network Working Group, October 2003.

[11] J.Leguay, V.Conan and T.Friedman: "QoS Routing in OLSR with Several Classes of Service", The 4th Annual IEEE International Conference on Pervasive Computing and Communications Workshops 2006 (PerCom Workshops 2006), pp. 420-425, March 2006.

[12] A.Amani, Y.Fakhri and J.Abouchabak: "QoS Routing and Performance Evaluation for Mobile Ad Hoc Networks Using OLSR Protocol", International Journal of Ad hoc Sensor and Ubiquitous Computing (IJASUC), Vol.2, No.2, June 2011.

[13] K. Kunavut and T. Sanguankotchakorn: "QoS-aware Routing for Mobile Ad Hoc Networks Based on Multiple Metrics: Connectivity Index (CI) and Delay", The 7th International Conference on Electrical Engineering/Electronics Computer Telecommunications and Information Technology 2010 (ECTICON 2010), pp. 46-50, May 2010.

[14] K. Kunavut and T. Sanguankotchakorn: "Multi-Constrained Path (MCP) QoS routing in OLSR Based on Multiple Additive QoS Metrics", International Symposium on Communications and Information Technologies 2010 (ISCIT 2010), pp. 226-231, October 2010. 
International Journal of Computer Networks \& Communications (IJCNC) Vol.10, No.6, November 2018

[15] K.Kunavut and T.Sanguankotchakorn:"Generalized Multi-constrained Path (G_MCP) QoS Routing for Mobile Ad Hoc Networks", Journal of Communications, Vol.7, No.3, pp.246-257, March 2012.

[16] Ram S. Dahal and T. Sanguankotchakorn: "QoS Routing in MANET through Cross-Layer Design with BER and Modifying AODV", Asian Himalayan International Conference on Internet (AHICI2011), The next Generation of Mobile, Wireless and Optical Communication Networks, Kathmandu, Nepal, November 4-6, 2011.

[17] H.Badis, A.Mavaretto, K.A.Agha and G.Pujolle, "Optimal Path Selection in a Link State QoS Routing Protocol", Proceeding of IEEE Vehicular Technology Conference, Vol.4, pp.2570-2574, May 2004.

[18] GeYing, T.Kunz and L. Lamont: "Quality of Service Routing in Ad-hoc Networks using OLSR", Proceedings of the 36th Annual Hawaii International Conference on System Sciences 2003,pp. 9, January 2003.

[19] X. Yuan, "Heuristic Algorithms for Multi-Constrained Quality-of-Service Routing", IEEE/ACM Transactions on Networking, Vol.10, Issue 2, pp.244-256, 2002.

[20] P.Khadivi, S.Samavi and T.D. Todd," Multi-constraint QoS Routing using a new Single Mixed Metrics", Journal of Network and Computer Applications, Vol.31, Issue 4,pp.656-676, 2008.

[21] Dong-won Shin, Edwin K.P. Chong and Howard Jay Siegel, "Multi-Post path-based Look ahead Multi-Constraint QoS Routing", Journal of the Franklin Institute, Vol.349, Issue 3, pp.1106-1124, 2012.

[22] Jiann-Liang Chen, Shih-Wei Liu, SZu-Lin Wu and Ming-Chiao Chen, "Cross-layer and Cognitive QoS Management System for Next Generation Networking", International Journal of Communication Systems, Vol. 24, pp.1150-1162, 2011.

[23] Min Chen, Liang Zhou, Takahiro Hara, Yang Ziao and Victor C.M. Leung", Advances in Multimedia Communications", International Journal of Communication Systems, Vol.24, pp.1243-1245, 2011.

[24] Li-Feng Zhou, Lei Chen, Hung Keng Pung and Lek Heng Ngoh, "Identifying QoS Violations through Statistical End-to-End Analysis" ,International Journal of Communication Systems, Vol.24, pp.13881406, 2011.

[25] Jin Ye, Jian-xin Wang and Jia-wei Huang, "A Cross-layer TCP for providing Fairness in Wireless Mesh Network", International Journal of Communication Systems, Vol.24, pp.1161-1626, 2011.

[26] Qiong Shi, Cristina Comaniciu, Dandan Wang and UfukTureli, "Cross-layer MAC Design for Location aware Wireless Sensor Networks", International Journal of Communication Systems, Vol. 24, pp.872-888, 2011.

[27] Weifeng Sun, Tong Fu, Feng Xia, Zhenquan Qin and Rong Cong, "A Dynamic Channel Assignment Strategy based on Cross-layer Design for Wireless Mesh Networks", International Journal of Communication Systems, Vol. 25, pp.1122-1138, 2012.

[28] Kun Yang, Xueqi Cheng and Giovanni Pau, "Interdisciplinary and Cross-layer Design of Mobile Social Networks and Wireless Networks", International Journal of Communication Systems, Vol.25, pp.1243-1244, 2012.

[29] T. Sanguankotchakorn, S. Shrestha and N.Sugino, "Effective Social Ad Hoc Networking on OLSR MANET using Similarity Approach", The 5th International Conference in Internet and Distributed Computing Systems (IDCS2012), pp.15-28, November 2012.

[30] V. Srivastava. and M. Motani: "Cross-Layer Design: A Survey and the Road Ahead", IEEE Communications Magazine, Vol.43, No.12, pp. 112-119, December 2005. 
[31] Marco Fotino and Floriano De Rango, "Energy Issues and Energy aware Routing in Wireless Ad-hoc Networks", http://www.intechopen.com/books/mobile-ad-hoc-networks-protocol-design/energyissues-and-energy-aware-routing-in-wireless-ad-hoc-networks ", pp.281-296, 2011.

[32] A.Kumar and Q.M.Rafiq and K.Bansal, "Performance Evaluation of Energy Consumption in MANET", International Journal of Computer Application, Vol .42, No.2, pp.7-12, March 2012.

[33] Jun Huang, Xiaohong Huang, Yan Ma, "Routing with Multiple Quality-of-Services Constraints: An Approximation Perspective", Journal of Network and Computer Applications, Vol. 35, Issue 1, pp. 469-479, 2012.

[34] Kirti Aniruddha Adoni and Radhika D. Joshi, "Optimization of Energy Consumption for OLSR Routing Protocol in MANET”, International Journal of Wireless and Mobile Networks, Vol.4, No.1, pp.251-262, February 2012.

[35] Adel Aneiba and Mohammed Melad, "Performance Evaluation of AODV, DSR, OLSR, and GRP MANET Routing Protocols Using OPNET", International Journal of Future Computer and Communication, Vol.5, No.1, pp. 57-60, February, 2016

[36] K.Kunavut and T.Sanguankotchakorn," Performance Evaluation of Ad Hoc Routing Protocols to Deliver MPEG-4 Traffic", The 12th IEEE International Conference on Communication Technology (ICCT2010), pp.207 -210,November 2010.

[37] W.Xiuchao, "Simulate 802.11b Channel within NS2", Technical Report, URL: http://read.pudn.com/downloads165/doc/756173/Simulate_802.11b_Channel_NS2.pdf, NUS,2004.

[38] Fall, K. and Varadhan, K: "Formerly NS Notes and Documentation", UC Berkeley, USC/ISI and Xerox PARC, November, 2011.

[39] The Network Simulator-ns- 2, http://www.isi.edu/nsnam/ns

[40] C.-H.Ke, and C.-K.Shieh, W.-S. Hwang, and A. Ziviani," An Evaluation Framework for More Realistic Simulations of MPEG Video Transmission", Journal In Information Science and Engineering, pp.425-440, 2008.

\section{Authors}

Teerapat Sanguankotchakorn was born in Bangkok, Thailand on December 8, 1965. He received the B. Eng in Electrical Engineering from Chulalongkorn University, Thailand in 1987, M. Eng and D. Eng in Information Processing from Tokyo Institute of Technology, Japan in 1990 and 1993, respectively. In 1993, he joined Telecommunication and Information Systems Research Laboratory at Sony Corporation, Japan where he holds two patents on Signal Compression. Since October 1998, he has been with Asian Institute of Technology where he is currently an

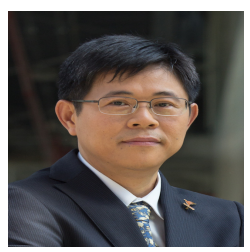
Associate Professor at Telecommunications Field of Study, School of Engineering and Technology. He is a Senior member of IEEE and member of IEICE, Japan.

Sanika K. Wijayasekara was born in Sri Lanka on January 14, 1986. She received her B.Sc.(Hons) in IT specialized in Computer System and Networking degree from Sri Lanka Institute of Information Technology, Sri Lanka in 2010 and M.Sc in Telecommunications from Asian Institute of Technology, Thailand in 2012. Her current research interests are in the area of Cross-Layer designs, QoS assurances in multimedia applications and wireless network protocols.

Nobuhiko Sugino was born in Yokkaichi, Mie, Japan on November 19, 1964. He received B. Eng, M. Eng, and D.Eng. in Physical Electronics from Tokyo Institute of Technology in 1987, 1989 and 1992, respectively. Since 1992, he has been with Tokyo Institute of Technology, where is now an Associate Professor at Department of Information System, Interdisciplinary Graduate School of Science and Engineering. Dr. Nobuhiko Sugino is a member of IEICE and IEEE. 